( $) 2018$, The Authors. Published by FASS Inc. and Elsevier Inc. on behalf of the American Dairy Science Association ${ }^{\circledR}$. This is an open access article under the CC BY-NC-ND license (http://creativecommons.org/licenses/by-nc-nd/4.0/).

\title{
Indicators of resilience during the transition period in dairy cows: A case study
}

\author{
I. D. E. van Dixhoorn, ${ }^{* 1}$ R. M. de Mol, ${ }^{*}$ J. T. N. van der Werf, ${ }^{*}$ S. van Mourik, $†$ and C. G. van Reenen* \\ *Livestock Research, and \\ †Farm Technology Group, Wageningen UR, Wageningen, the Netherlands 6708 WD
}

\section{ABSTRACT}

The transition period is a demanding phase in the life of dairy cows. Metabolic and infectious disorders frequently occur in the first weeks after calving. To identify cows that are less able to cope with the transition period, physiologic or behavioral signals acquired with sensors might be useful. However, it is not yet clear which signals or combination of signals and which signal properties are most informative with respect to disease severity after calving. Sensor data on activity and behavior measurements as well as rumen and ear temperature data from 22 dairy cows were collected during a period starting 2 wk before expected parturition until 6 wk after parturition. During this period, the health status of each cow was clinically scored daily. A total deficit score (TDS) was calculated based on the clinical assessment, summarizing disease length and intensity for each cow. Different sensor data properties recorded during the period before calving as well as the period after calving were tested as a predictor for TDS using univariate analysis of covariance. To select the model with the best combination of signals and signal properties, we quantified the prediction accuracy for TDS in a multivariate model. Prediction accuracy for TDS increased when sensors were combined, using static and dynamic signal properties. Statistically, the most optimal linear combination of predictors consisted of average eating time, variance of daily ear temperature, and regularity of daily behavior patterns in the dry period. Our research indicates that a combination of static and dynamic sensor data properties could be used as indicators of cow resilience.

Key words: dairy cow, transition period, resilience, dynamic indicator, behavior

Received March 20, 2018.

Accepted July 13, 2018.

${ }^{1}$ Corresponding author: ingrid.vandixhoorn@wur.nl

\section{INTRODUCTION}

The transition period, defined as the period between 3 wk prepartum and 3 wk postpartum (Grummer, 1995), is a demanding period for dairy cows. It makes them vulnerable to the development of metabolic and infectious diseases, especially in the first weeks after calving (Urton et al., 2005; Huzzey et al., 2007). Incidences of common diseases such as milk fever, clinical respiratory diseases, contagious mastitis, and clinical parasitism have been reduced in the last $25 \mathrm{yr}$ due to the shift in focus on disease prevention rather than treatment, but the incidence of important periparturient diseases remains high (LeBlanc et al., 2006). It has been argued that to reduce the incidence of periparturient diseases we should look at the farm as an integrated system rather than rely on growing knowledge on pathology and etiology (LeBlanc et al., 2006). In particular, the existence of complex relationships between periparturient diseases and nutritional strategies, housing conditions, and social and attitudinal factors is believed to hamper effective prevention and control (Mulligan and Doherty, 2008). Therefore, early warning signals for disease and the identification of farm-specific risk factors remain key elements for opportune interventions.

Metabolic and inflammatory response measures have been described as early indicators for periparturient diseases, providing opportunities for early treatment (Huzzey et al., 2009, 2011, 2015; Seifi et al., 2011; Roberts et al., 2012; Trevisi et al., 2012). Preferably, indicators are used in farm management even before the onset of disease becomes apparent. For this reason, research should also focus on the detection of cows that are statistically more likely to develop disease in addition to early disease detection. Detection of cows at risk would allow for adequate preventive intervention, often without the need for medical treatment. There is growing interest in how to use sensor data to detect cows at risk for periparturient disorders (Weary et al., 2009; Rutten et al., 2017). Indeed, automatically recorded behaviors have shown to be indicative for risk of disease (Weary et al., 2009). For example, reduced 
feed intake during the prepartum transition phase was proven to be an indicator for cows at risk for metritis (Urton et al., 2005; Huzzey et al., 2007). Average levels of rumination, feeding, and other behaviors during the transition period differ between sick and healthy cows and were suggested as early indicators for periparturient disorders such as metritis and clinical or subclinical ketosis (Urton et al., 2005; Huzzey et al., 2007; Goldhawk et al., 2009; Calamari et al., 2014). Average levels of feeding, rumination, and other behaviors can easily be acquired noninvasively using automated and highfrequency sensor measurements. Moreover, compared with human observers, sensors are often more sensitive or less biased (Weary et al., 2009; Rutten et al., 2017). Average levels of sensor data output are commonly calculated as representative signal property. When looking only at average levels of sensor-generated time series, useful information on underlying mechanisms may get lost. Dynamic properties of time series generated by sensors may contain extra useful information (Peng et al., 2009). Based on these outcomes, we hypothesized that combining sensors and multiple signal properties per sensor can improve prediction of disease length and severity during the transition period. We tested this hypothesis by predicting the total length and severity of transition-related diseases with different combinations of signal types and properties. The transition diseases are all interrelated, and the onset and appearance of these disorders may not always be the same (Mulligan and Doherty, 2008; Sundrum, 2015). We therefore did not focus on pathogenesis of one single disease but rather tried to find predictors of generic impaired health during this period independent of the onset, underlying pathway or specific disease. To quantify the prediction accuracy of total length and severity of transition-related diseases, we defined a total deficit score (TDS), which is a total score of postpartum clinical aberrations, acquired through daily clinical inspection by veterinarians. The higher the TDS, the less resilient the cows were to the challenge of transition when defining resilience in dairy cows as the capability to stay healthy during and after the demanding challenge of transition (modified from Colditz and Hine, 2016). We calculated prediction errors for TDS based on the dynamic patterns and averages of high-resolution, highfrequency physiological and behavioral data recorded in individual cows before calving. We also calculated prediction errors for TDS based on data recorded during the first week after calving.

\section{MATERIALS AND METHODS}

This study adhered to the established principles of laboratory animal use and the Dutch laws related to animal experiments. The Wageningen University Animal Care and Use Committee (Lelystad Department) approved the experiment under protocol number 2014039.b.

\section{Animals, Housing, and Diet}

A group of 22 Dutch Holstein-Friesian dairy cows of mixed age (13 primiparous, 9 multiparous) within a Dutch dairy farm situated in the east of the Netherlands was selected for the experiment. The experiment took place between April 14 and July 26, 2014. During this period, 26 cows were enrolled based on the expected day of parturition. Experimental period per cow lasted from $2 \mathrm{wk}$ before expected parturition until $6 \mathrm{wk}$ after parturition. Cows were included for analysis when they were scored as healthy during the dry period and when a complete clinical data set of the experimental period was available. Based on these criteria, 22 cows were selected. The cows were part of the herd of 180 cows, with an average production of $10,040 \mathrm{~kg}$ of milk (with $4.25 \%$ fat and $3.58 \%$ protein) per year. The lactating cows, dry cows, and pregnant heifers were housed in a freestall barn with cubicles. Bedding material in the cubicles consisted of a layer of approximately $5 \mathrm{~cm}$ of sawdust on the concrete floor. During the 2 wk before the expected day of parturition, dry cows and pregnant heifers were kept in 1 pen with 14 feeding places (with individual head locks), 15 cubicles, and 1 water point. Average group size was 15 cows (range: 14-16). On average, 1 or 2 cows were introduced and 1 or 2 cows were removed from this dry cow pen every week. When the cows showed signs of parturition, they were moved to an individual straw-bedded maternity pen within the same building. After calving, they were introduced into 1 of 3 pens with lactating cows. The 3 pens with lactating cows were each milked with a DeLaval (Tumba, Sweden) milking robot and were similar with regard to production level. Two of these 3 pens were of similar size, and to each group 6 experimental cows were introduced. In both pens, 40 to 42 cows had access to 60 cubicles and 35 feeding places with individual head locks. The third pen had 43 feeding places with individual head locks and 62 cubicles for 49 to 51 cows. Ten experimental cows were introduced into this third pen. All lactating cow pens had 3 drinking places. Number of cows per pen was kept as constant as possible, and cows remained in the same group after calving until the next dry period. Group composition was dynamic, as animals were moved between pens before and after the dry period. On average, every 2 wk 1 cow was introduced and 1 cow was removed from lactating cow pens. The cows were fed twice daily a TMR consisting of corn silage, hay silage, with added concentrates (protein and 
mineral supplement) adjusted to the production level of the group. Dry cows were fed a dry cow diet consisting of TMR. Water was available ad libitum. Feed composition was kept constant for the duration of the experimental period.

\section{Clinical Examination and Blood Sampling}

A score was calculated based on clinical examination of each cow as described by Hajer et al. (1988) that was performed daily for the period of $2 \mathrm{wk}$ before until 6 wk after parturition. All 22 cows were clinically scored daily during the complete period. During clinical examination the following aspects were scored: heart rate (beats per minute), breathing rate (breaths per minute), rectal temperature $\left({ }^{\circ} \mathrm{C}\right)$, rumination (chews per minute), BCS (Edmonson et al., 1989), locomotion score (according to the scoring system of D. Zaaijer, W. D. J. Kremer, and J. P. T. M. Noordhuizen in Hulsen, 2012), udder condition [per quarter: skin temperature (too warm, too cold, normal), color (red, abnormal, normal), painful during palpation (yes or no), swollen (yes or no), teat condition (flexible, color, painful)], retained placenta (RP; was diagnosed if the placenta was protruding from the vulva $24 \mathrm{~h}$ after calving), uterus condition and excretion (size of the uterus by rectal examination, color and smell of vaginal discharge, and the amount of pus and mucus), manure consistency (according to the scoring system of D. Zaaijer, W. D. J. Kremer, and J. P. T. M. Noordhuizen in Hulsen, 2012), possible displaced abomasum (by auscultation), and overall condition (healthy or not). Clinical examinations were performed by 3 specialized bovine practitioners, and the outcome was not shared with the farmer. The farmer followed his or her standard strategy to decide whether to treat a cow or call a veterinarian. If the cow was diagnosed as ill, treatment of the cow was recorded.

Blood samples were collected every $2 \mathrm{~d}$ from the coccygeal vein into $10-\mathrm{mL}$ sterile serum tubes (Vacutainer, Becton Dickinson, Franklin Lakes, NJ). The blood was centrifuged (1-4 h after collection at room temperature at 1,438 $\times g$ for 7-10 min) and the serum was separated into several aliquots and stored at $-20^{\circ} \mathrm{C}$ until analysis. Blood values indicative and related to periparturient problems were determined to corroborate our clinical assessment and consisted of calcium, inorganic phosphorus, albumin, haptoglobin, urea, and nonesterified fatty acids (Seifi et al., 2011; Roberts et al., 2012; Trevisi et al., 2012). Analyses were performed at a commercial laboratory (GD Animal Health, Deventer, the Netherlands). To evaluate potential farm factors influencing cow performance, a risk analysis and assessment of the quality of housing and management at farm level was performed 6 times during the experimental period using the Dutch farm monitoring tool KoeKompas (CowCompas; https://zuivelplatform .nl) in which the individual farm is scored on 7 critical success factors, 40 performance indicators, and more than 100 management control points. This assessment was performed by a specially trained veterinarian who was not involved in the clinical examinations and who was unaware of any other results of the current experiment. Based on the assessment, the veterinarian wrote a qualitative report in which the most critical management risks present at the moment the assessment was performed were described.

\section{Postpartum TDS}

Each aberrant clinical finding or disease-related symptom was scored daily as 1 . Deficits were scored as 1 when clinical findings were above or below normal values as described by Hajer et al. (1988). Diseaserelated symptoms were divided into mastitis-related problems (score 1 if 1 or all of the features were found positive: swollenness, painfulness, or redness), metritisrelated problems (score 1 if 1 or all of the features were found positive: RP, abnormal vaginal discharge, or large uterus), lameness-related problems (score 1 if locomotion score was 5 or when diagnosed lame), and other health problems (fever, manure score 1 or 5 , dull cows, no rumen activity, not eating, milk fever, treated by farmer). All daily clinically detected deficits were added to 1 total score (dimensionless) during the 6 -wk period after calving. This resulted in 1 total TDS per cow based on clinical findings; blood values were not included in the TDS. By adding daily scores of clinical aberrations, the length of the disease-related problems was also taken into account in the TDS. Cows with a low TDS with few aberrant clinical findings lasting only a short period of time (fast recovery) were in good health, whereas cows with a high TDS suffered from more health problems or problems with longer duration (slow recovery) during the 6 -wk period after calving. We first evaluated whether our TDS was distinctive for impaired health using blood concentrations associated with metabolic stress and other periparturient disorders as a reference. For this evaluation, we distinguished 3 groups: group 1 with scores of TDS $<7$ (good health), group 2 with TDS 7 to 14 (intermediate health), and group 3 with TDS $>20$ (bad health). These arbitrary thresholds were chosen to obtain similar numbers of cows per group. The area under the curve (AUC) of blood values of each individual cow was calculated for 2 separate periods using the linear trapezoidal method. 
The following 2 periods were defined for the TDS evaluation: from $10 \mathrm{~d}$ before until $1 \mathrm{~d}$ before parturition (antepartum) and from parturition until $36 \mathrm{~d}$ after parturition (postpartum). The AUC before parturition and after parturition of the different blood values were averaged per TDS group 1, 2, and 3 and compared by pairwise comparison. The AUC of the period before parturition was used to evaluate the health state during the dry period, and the AUC of the period after parturition was used to evaluate the TDS.

\section{Sensor Data Acquisition}

During the 2 -wk period before calving and the $6 \mathrm{wk}$ after calving, continuous and high-frequency behavioral and body temperature data were obtained with the use of 3 sensors that previously had been validated (Bewley and Schutz, 2010; Borchers et al., 2016):

1. IceQube sensors (IceRobotics, Edinburgh, UK) were used for recording activity: per quarter the number of minutes lying and standing (adding up to 15), number of steps, number of lying bouts, and motion index (a measure of the animal's activity level; Munksgaard et al., 2006). IceQube sensors were attached at least 2 wk before expected parturition date.

2. SensOor sensors (Agis Automatisering, Harmelen, the Netherlands) were used for measuring behavior (eating, ruminating, and activity level) and ear temperature (Bikker et al., 2014). Time each hour was divided into number of minutes eating, ruminating, highly active, low active, and inactive (determined by classifying algorithms; see Pereira et al., 2018). Average ear temperature $\left({ }^{\circ} \mathrm{C}\right)$ per hour was also recorded. A 3-dimensional accelerometer continuously registered the movements of the cow's ear. SensOor sensors were attached in a later stage of the experiment. SensOor data were obtained for only 7 cows during the entire 2 wk before parturition and for 10 cows during the first week after parturition.

3. BellaAg Bolus sensors (Wynnstay, Powys, UK) were used for measuring rumen temperature $\left({ }^{\circ} \mathrm{C}\right)$ every 10 min (Timsit et al., 2011). BellaAg Bolus sensors were inserted in the rumen at least $2 \mathrm{wk}$ before expected parturition date.

\section{Data Analysis and Statistical Analysis}

IceQube data obtained per cow per 15 min were summed to get data at hour level, and BellaAg bolus temperature data per cow per 10 min were averaged per hour. Standing time of the IceQube data was excluded from the analysis because it was directly complementary to lying time. Average, variance, autocorrelation (with lag $\tau=1 \mathrm{~h}$ ), and nonperiodicity were calculated per cow for each hourly sensor variable. Sensor variables were obtained during 2 periods: the dry period, starting $15 \mathrm{~d}$ before calving up to and including the day before calving (referred to as long-term), and the period after calving from d 1 up to d 7 after calving (referred to as short-term). For both periods and each sensor variable, the average, variance, and autocorrelation were calculated over all measurement values. Variance and autocorrelation are indicative of the amount of variation in behavior and other variables and of the level of consistency or regularity of the behavior. For this research, nonperiodicity was introduced as a measure of regularity in the daily pattern of the sensor data and was defined as the mean squared error of the correlogram with a sinusoid with a 24-h cycle and an amplitude of 0.25 (Figure 1). The nonperiodicity was calculated over the same 2 periods. Univariate analysis of covariance was performed with cow as experimental unit, with TDS as dependent variable, and with each sensor-based metric (i.e., average, variance, autocorrelation, and nonperiodicity, before and after parturition) as an explanatory variable. Parity was included in the univariate analysis of covariance model as a fixed effect with 2 levels (i.e., parity 1, parity 2 or higher). Prior to analysis of covariance, we examined the fixed effects of lactating cow pen (pen 1, 2, or 3) on both TDS and sensor-based explanatory variables with ANOVA. Because no significant effects of pen were found, lactating cow pen was not considered in subsequent analyses of covariance. The TDS was log-transformed for the analysis to $\log (1+$ TDS $)$, which led to less skew distribution and a better model fit. We made a distinction between predictors before calving (long-term predictors) and predictors after calving (short-term predictors). Statistical significance was assumed if $P \leq 0.05$. Only significant results and tendencies $(P<0.10)$ are shown in the results. Next, a multivariate step was performed using the significant predictors and a selection procedure (best subset selection with the "regsubsets" function from the R library "leaps") to obtain a prediction model with an optimal subset of predictors (James et al., 2013). Finally, a leave-one-out cross-validation (LOOCV; James et al., 2013) was performed. For the LOOCV, each observation is used successively as a validation set with the remaining observations as the training set. The resulting LOOCV estimate is the mean squared error (MSE) of the single MSE. The multivariate model was built by applying best subset selection on all significant explanatory variables (non- 

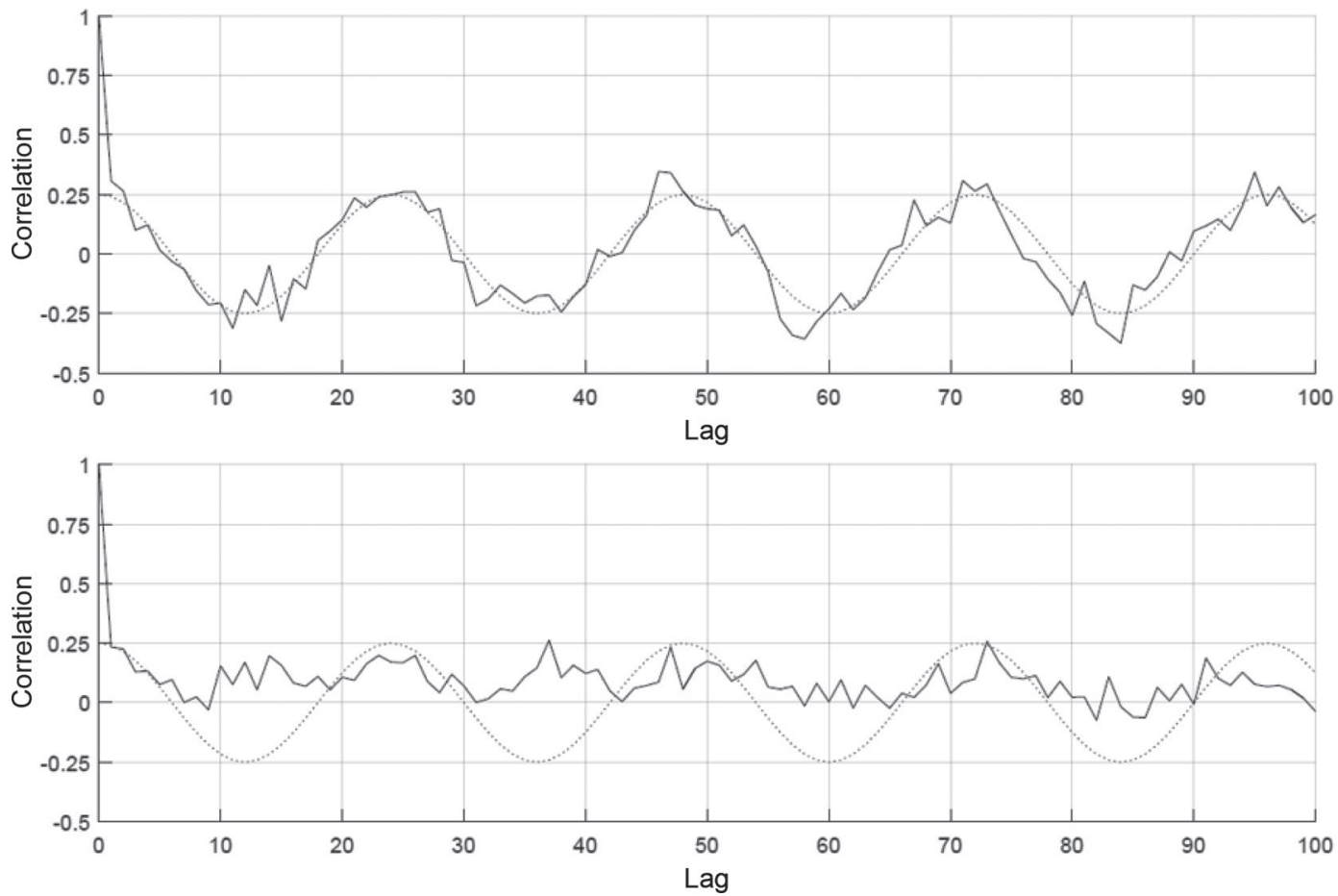

Figure 1. Examples of nonperiodicity, a measure of the regularity in the daily pattern of the sensor data and defined as the mean squared error of the correlogram (black line) with a sinusoid with a 24-h cycle and an amplitude of 0.25 (dotted line). Top: cow 1 with low (0.005) nonperiodicity for eating time; (bottom) cow 2 with high (0.034) nonperiodicity for eating time.

periodicity of motion index was excluded because it is known to be correlated with the number of steps; de Mol et al., 2013). The results may be influenced by the relative low number of cases, as only 7 out of 22 had data of all sensors acquired during the complete measuring period. These 7 cows had a TDS of $0,6,10$, $14,21,72$, and 121 , which we tentatively considered as a representative distribution of TDS. When only applying the 12 IceQube explanatory variables, all 22 cases could be used. Therefore, an additional analysis was done applying only the IceQube variables based on lying time, number of steps, and number of lying bouts as input variables. For the additional analysis, again best subset selection was applied with LOOCV using the "regsubsets" function from the R library "leaps." All analyses were performed with the statistical programming language R (R Core Team, 2017).

\section{RESULTS}

\section{Representativeness of TDS for Disease Severity}

Clinical observations were summarized into a TDS per cow after the transition period. The TDS values per cow varied between 0 and 121. Six cows had a TDS smaller than 7 (group 1), 7 cows had a TDS between 7 and 14 (group 2), and 9 cows showed a score larger than 20 (group 3). No displaced abomasum was diagnosed, and 1 cow was treated by the farmer for milk fever after calving. This same cow was given analgesics, was systemically treated with antibiotics (treating metritis), and had the highest TDS. Five other cows were treated by the farmer, 2 cows with analgesics due to parturition problems ( 1 with uterus torsion and 1 with vaginal injuries), 1 cow with analgesics and intramuscular oxytetracycline treatment for endometritis, and 2 cows were treated locally between the claws for mortellaro. Mastitis-related symptoms were recorded in 11 cows for more than $3 \mathrm{~d}$, and 8 cows showed metritis-related symptoms for more than $3 \mathrm{~d}$. Lameness was recorded in 7 cows for more than 3 d. General illness was recorded in 4 cows ( 1 cow for $1 \mathrm{~d}, 1$ cow for $5 \mathrm{~d}$, and 2 cows for 37 and $39 \mathrm{~d}$, respectively). Averages per TDS group for the different blood values are plotted in Figure 2 . In the first period, before parturition, no differences were detected between the 3 groups in any of the blood parameters, which was in line with our clinical findings. The 3 TDS groups started to diverge in all blood values except nonesterified fatty acids and urea after parturition when clinical aberrations started to evolve. In the period after parturition, group 3 showed the slowest recovery in calcium and albumin and an elongated peak 
in haptoglobin (Figure 2). In the analysis of the period after parturition, the AUC for calcium in group 1 was significantly higher compared with group $3(P<0.01)$ and tended to be higher compared with group $2(P$ $<0.10$ ). For group 2, AUC for calcium tended to be higher compared with group $3(P=0.06)$. The AUC for phosphorus in group 3 tended to be lower compared with groups $1(P=0.1)$ and $2(P<0.05)$. The AUC for albumin in group 1 was higher compared with groups $2(P<0.05)$ and $3(P<0.001)$, and group 2 tended to be higher compared with group $3(P=0.07)$. For haptoglobin, differences were present between groups 1 and $2(P<0.10)$ and between groups 1 and $3(P<$
0.001). We also found that an increase in haptoglobin and a decrease in calcium preceded clinical aberrations (results not shown).

\section{Qualitative Evaluation of the Risk Assessment CowCompass}

In the risk assessment analysis ÇowCompass (KoeKompas), overstocking (113 feeding places for 165 cows) in combination with insufficient access to important resources such as feed, water, and cubicles was repeatedly identified as an important risk factor at the farm where we performed the study.

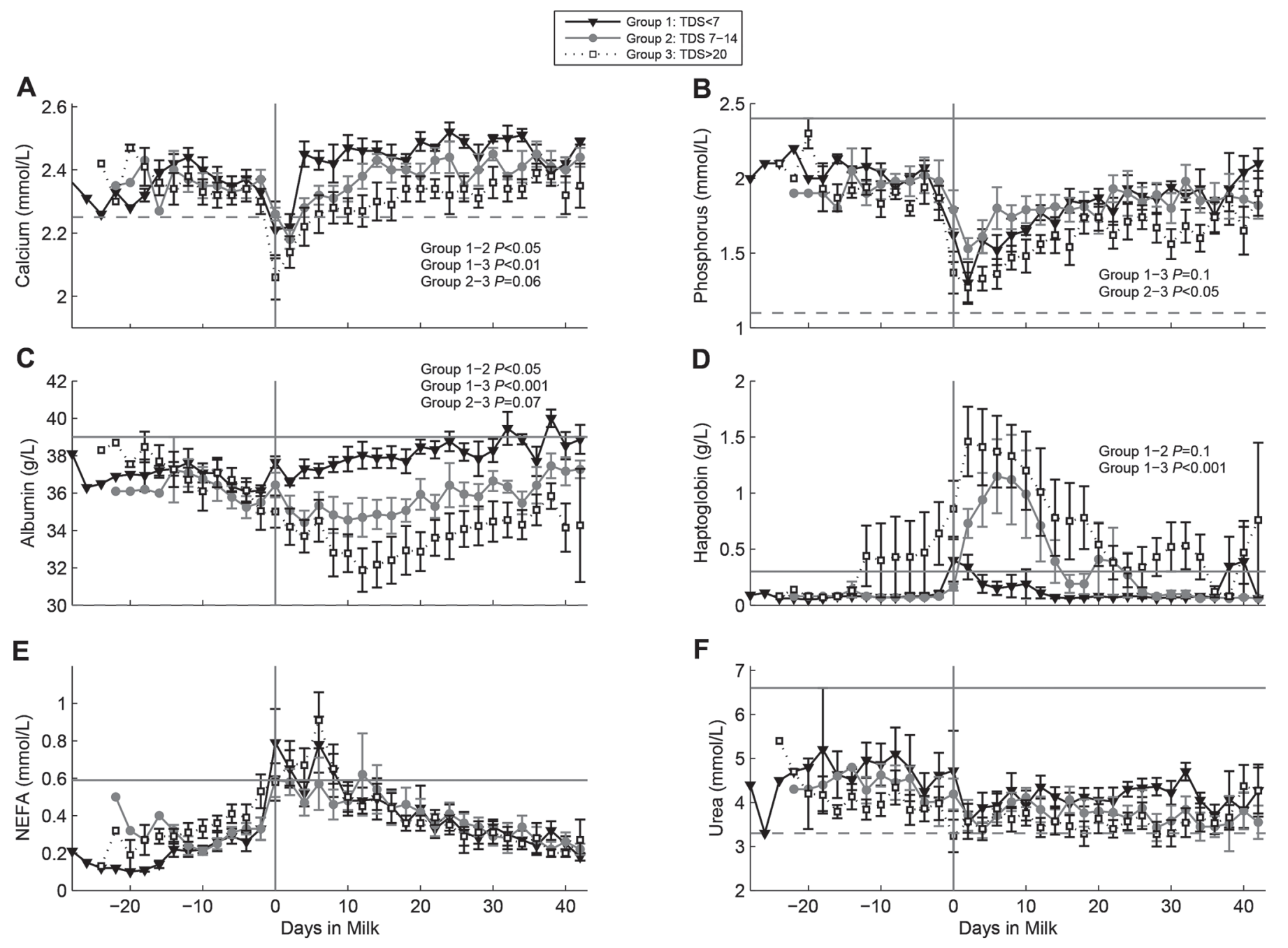

Figure 2. Patterns of different serum concentrations (mean per group \pm SEM) per total deficit score (TDS) group for (A) calcium (mmol/L), (B) phosphorus (mmol/L), (C) albumin $(\mathrm{g} / \mathrm{L}),(\mathrm{D})$ haptoglobin $(\mathrm{g} / \mathrm{L}),(\mathrm{E})$ nonesterified fatty acids $(\mathrm{mmol} / \mathrm{L})$, and $(\mathrm{F})$ urea $(\mathrm{mmol} / \mathrm{L})$. Black line with downward facing triangle marker: group 1 (TDS $<7$ ); gray line with circle marker: group 2 (TDS 7-14); dotted line with square marker: group 3 (TDS >20). Gray vertical line at DIM $=0$ indicates the day of parturition. Gray horizontal line indicates upper reference value; gray dotted horizontal line indicates lower reference value. Significant differences of area under the curve between TDS groups after parturition are indicated in the graphs. 


\section{Long-Term Predictors (Recorded Before Calving) of Postpartum TDS}

We first evaluated the sensor data properties that were acquired before parturition (long-term) as predictors for TDS after calving. Significant relations were found between $\log (1+$ TDS $)$ and eating time and between $\log (1+$ TDS $)$ and the variance in ear temperature. Nonperiodicity of eating and lying time, number of steps, and motion index especially showed significant relations with $\log (1+$ TDS $)$ (Table 1$)$.

The multiple regression model resulted in 3 longterm predictors: average eating, variance ear temperature, and nonperiodicity of number of steps (Figure 3). The training MSE was 0.0279 based on all complete observations $(\mathrm{n}=7)$. Then, the MSE was calculated by LOOCV on a multiple linear regression model with the 3 selected explanatory variables as predictors; this resulted in an average test prediction error (MSE) of 0.188 (TDS). If restricted to 1 predictor, the MSE increased to 0.0338 . The test MSE when using only 1 predictor decreased to 0.0948 .

Best subset selection of the additional analysis applying only the 12 IceQube explanatory variables (based on lying time, number of steps, and number of lying bouts as input variables) resulted in 4 long-term predictors: average number of lying bouts, variance lying time, nonperiodicity lying time, and nonperiodicity number of lying bouts with MSE $=0.0551$ (MSE $=$ 0.112 if restricted to 1 predictor; Figure 3). Estimating the MSE by LOOCV resulted in an average MSE of 0.112 . The test MSE when using only 1 predictor increased to 0.140 .

\section{Short-Term Predictors (Recorded After Calving) of Postpartum TDS}

For the short-term predictors for TDS, we evaluated the sensor data properties that were acquired during the first week after parturition. For 9 explanatory variables, significant relations between $\log (1+$ TDS $)$ and sensor quantities were found (Table 2). In comparison with the prepartum period (long-term predictors), the number of significant explanatory variables was higher for sensor data recorded after calving (short-term predictors).

The multivariate model resulted in a multiple regression model with 4 short-term predictors: average number of steps, variance number of steps, autocorrelation lying time, and nonperiodicity eating. Based on all complete observations $(\mathrm{n}=10)$, the training MSE was 0.0101 (MSE $=0.0861$ if restricted to 1 predictor $)$. The MSE calculated by LOOCV on a multiple linear regression model with the 4 selected short-term explanatory variables as predictors was 0.0367 . The test MSE when using only 1 predictor increased to 0.227 .

\section{DISCUSSION}

\section{Representativeness of TDS for Disease Severity}

Slow recovery in calcium and albumin and an elongated peak in haptoglobin after parturition are all indicative of periparturient problems (Seifi et al., 2011; Roberts et al., 2012; Trevisi et al., 2012). Among the 3 groups of cows categorized according to TDS, cows with the highest TDS (group 3) showed the slowest

Table 1. Unstandardized estimates $(\beta)$ of long-term explanatory variables recorded before calving for $\log (1+\operatorname{TDS})^{1}$

\begin{tabular}{|c|c|c|c|c|c|c|c|}
\hline \multirow[b]{2}{*}{ Calculation } & \multirow[b]{2}{*}{ Sensor } & \multirow[b]{2}{*}{ Feature } & \multirow[b]{2}{*}{ No. } & \multirow[b]{2}{*}{ Intercept } & \multicolumn{3}{|c|}{ Unstandardized coefficient } \\
\hline & & & & & $\beta$ & $\mathrm{SE}$ & $P$-value \\
\hline \multirow[t]{3}{*}{ Average } & SensOor $^{2}$ & Inactive $(\mathrm{min} / \mathrm{h})$ & 7 & -0.59 & 0.09 & 0.04 & 0.07 \\
\hline & SensOor & Eating $(\min / \mathrm{h})$ & 7 & 3.24 & -0.16 & 0.02 & $<0.01$ \\
\hline & IceQube $^{3}$ & Motion index $(-)$ & 17 & 2.53 & -0.007 & 0.004 & 0.08 \\
\hline \multirow[t]{3}{*}{ Variance } & SensOor & Eating $(\mathrm{min} / \mathrm{h})$ & 7 & 2.75 & -0.009 & 0.004 & 0.06 \\
\hline & SensOor & Ear temperature $\left({ }^{\circ} \mathrm{C}\right)$ & 7 & -0.99 & 0.79 & 0.19 & $<0.05$ \\
\hline & IceQube & Steps (no.) & 17 & 1.74 & $-4 \times 10^{-4}$ & $2 \times 10^{-4}$ & 0.09 \\
\hline \multirow[t]{5}{*}{ Nonperiodicity } & SensOor & Eating (min/h) & 7 & -0.02 & 63.40 & 17.60 & $<0.05$ \\
\hline & SensOor & Ear temperature $\left({ }^{\circ} \mathrm{C}\right)$ & 7 & 0.09 & 7.33 & 3.04 & 0.07 \\
\hline & IceQube & Lying time $(\min / 15 \mathrm{~min})$ & 17 & 0.44 & 49.81 & 9.66 & $<0.001$ \\
\hline & IceQube & Steps (no.) & 17 & 0.60 & 42.20 & 14.10 & $<0.05$ \\
\hline & IceQube & Motion index $(-)$ & 17 & 0.50 & 45.70 & 15.80 & $<0.05$ \\
\hline
\end{tabular}

\footnotetext{
${ }^{1} \mathrm{TDS}=$ total deficit score
}

${ }^{2}$ Agis Automatisering (Harmelen, the Netherlands).

${ }^{3}$ IceRobotics (Edinburgh, UK). 
recovery of calcium and albumin, and cows with the lowest TDS (group 1) showed the fastest recovery of calcium and albumin levels after calving. The patterns of the blood constituents over time strongly support the idea that our TDS was representative of the degree and duration of diminished health. The blood results also confirm that the cows could be classified as healthy before parturition. Our TDS was based on clinical examinations only; additional features could be included (e.g., BHB measured in blood), and possible additional value should be evaluated in future studies.

\section{Average Levels of Single Predictors}

Our study identified different statistically significant explanatory variables for TDS before (long-term predic- tors) and after (short-term predictors) calving. Feeding, ruminating time, and DMI are usually reduced before parturition and continue to decline after calving when compared with baselines (Schirmann et al., 2013), but this decline is especially larger in sick animals suffering ketosis, metritis, or other health problems (Urton et al., 2005; Huzzey et al., 2007; Schirmann et al., 2016). The decline in feeding, ruminating time, and DMI before parturition is especially larger in animals suffering ketosis, metritis, or other postparturition problems as previously described (Urton et al., 2005; Huzzey et al., 2007; Schirmann et al., 2016). Goldhawk et al. (2009) also showed that healthy animals showed higher DMI, visited the feeder more often, and spent more time at the feeder during the dry period. The present relations between average signal levels of feeding time and TDS,
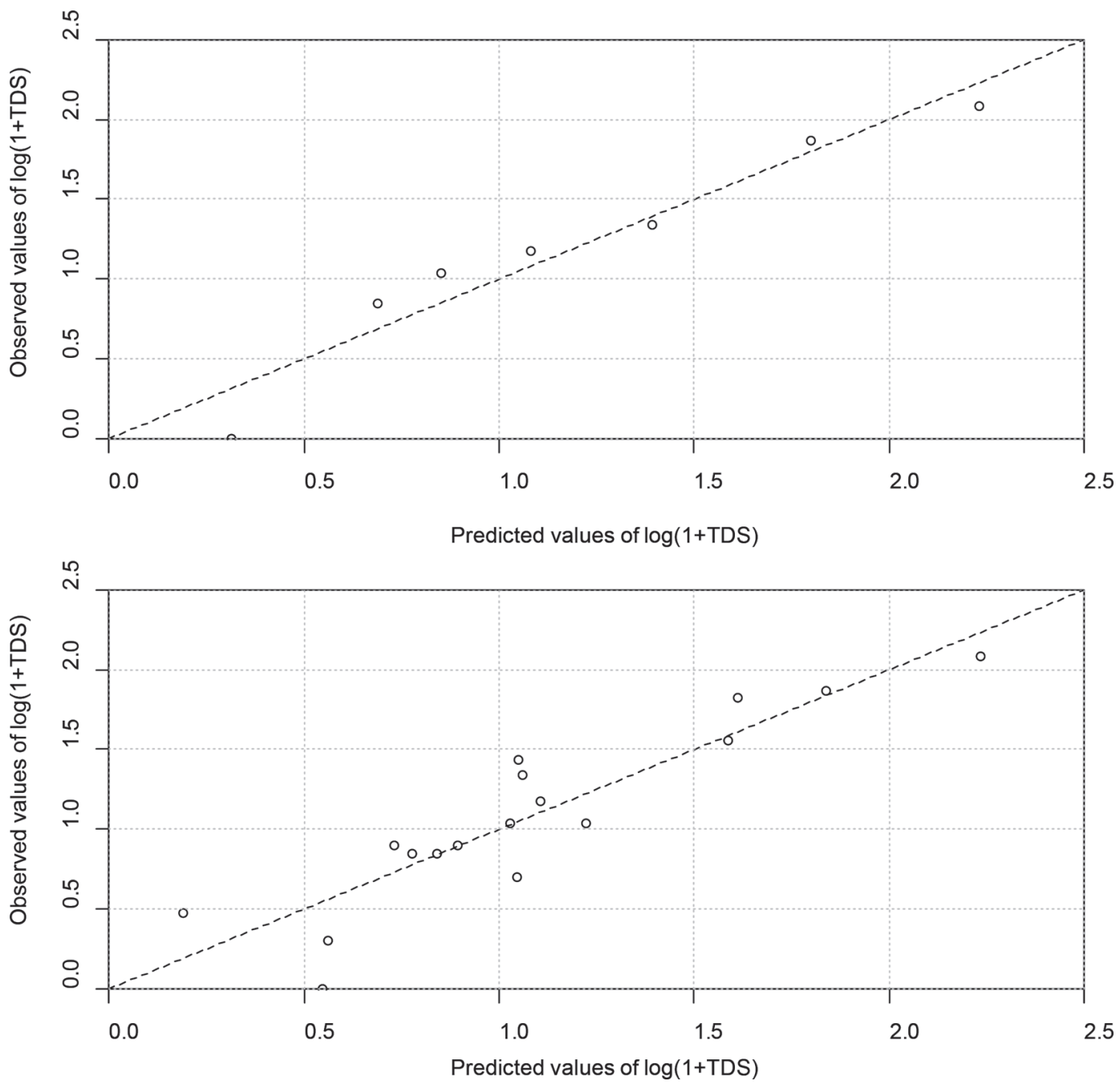

Figure 3. Scatter plots of the observed versus predicted values of $\log (1+$ TDS $)$ found by best subset selection on (top) the 3 most significant variables out of 5 (average eating, variance ear temperature, and nonperiodicity of number of steps) and (bottom) the 4 most significant IceQube (IceRobotics, Edinburgh, UK) variables out of 12 (average number of lying bouts, variance of lying time, nonperiodicity of lying time, and nonperiodicity of lying bouts). TDS = total deficit score 
both before and after calving, are in agreement with previous studies documenting similar relations (Urton et al., 2005; Huzzey et al., 2007; Schirmann et al., 2016). We suggest that explanatory variables recorded after calving (short-term predictors) represent indicators for disease and that explanatory variables recorded before calving (long-term predictors) represent indicators for the risk of developing disease, as we did not find detectable clinical or hematological aberrations before calving. Our finding that several of the same statistically significant explanatory variables for TDS were obtained both before parturition and after calving (i.e., averages of motion index, inactive time, eating time, and variances of eating time and number of steps) could also mean that these variables recorded before calving may relate to the onset of illness before it is clinically or hematologically detectable (Weary et al., 2009).

\section{Dynamic Signal Properties as Single Predictors}

We observed considerable added value of using dynamic signal properties (variance, autocorrelation, and nonperiodicity) for disease prediction, including variances in ear and rumen temperature, eating time and number of steps, and autocorrelations in rumen temperature and lying time. These dynamic properties indicate how much variation is expressed in the signal of the longitudinally measured behavioral and other variables and how consistent or regular the behavior is.
The capacity of cows to show consistent and regular diurnal behavior and temperature patterns seemed to be of great value to smoothly undergo the transition phase. To quantitatively express this characteristic, we introduced nonperiodicity as a measure of the consistency and regularity of (daily) patterns. A consistent and regular diurnal behavior or temperature pattern is equivalent to a low nonperiodicity during the dry period, and vice versa. Thus, cows with a low TDS showed regular behavioral patterns, or low nonperiodicities, during the dry period, reflecting stable diurnal rhythms of these behaviors, whereas cows with serious health disorders after parturition exhibited the opposite. In mammals, daily rhythms in behavior and physiology are programmed by a hierarchical interconnection of biological clocks located throughout the brain and body, known as the circadian system (Evans and Davidson, 2013). Mounting evidence indicates that disruption of circadian regulation or misalignment in mammals is associated with a wide variety of adverse health consequences (Evans and Davidson, 2013) and that, conversely, the integrated circadian timekeeping system enhances health, wellness, and longevity (Davidson et al., 2006; Gillette, 2013). The role of circadian disruption is difficult to assess in disease disorders because the influence of potential confounding factors is difficult to unravel. What we do know is that disturbed behavior patterns frequently coincide with accelerated disease progression and severity (Gillette, 2013). This might be one of the reasons why nonperiodicity was associated with disease severity in the current study.

Table 2. Unstandardized estimates $(\beta)$ of short-term explanatory variables recorded after calving for $\log (1+\mathrm{TDS})^{1}$

\begin{tabular}{|c|c|c|c|c|c|c|c|}
\hline \multirow[b]{2}{*}{ Calculation } & \multirow[b]{2}{*}{ Sensor } & \multirow[b]{2}{*}{ Feature } & \multirow[b]{2}{*}{ No. } & \multirow[b]{2}{*}{ Intercept } & \multicolumn{3}{|c|}{ Unstandardized coefficient } \\
\hline & & & & & $\beta$ & $\mathrm{SE}$ & $P$-value \\
\hline \multirow[t]{5}{*}{ Average } & SensOor $^{2}$ & Inactive $(\mathrm{min} / \mathrm{h})$ & 10 & 0.18 & 0.05 & 0.02 & 0.09 \\
\hline & SensOor & Eating $(\min / \mathrm{h})$ & 10 & 1.98 & -0.08 & 0.03 & $<0.05$ \\
\hline & SensOor & Ear temperature $\left({ }^{\circ} \mathrm{C}\right)$ & 10 & 15.97 & -0.49 & 0.14 & $<0.05$ \\
\hline & IceQube $^{3}$ & Steps (no.) & 18 & 1.89 & -0.01 & 0.002 & $<0.001$ \\
\hline & IceQube & Motion index $(-)$ & 18 & 1.80 & -0.002 & $6 \times 10^{-4}$ & $<0.001$ \\
\hline \multirow[t]{4}{*}{ Variance } & SensOor & Eating $(\min / \mathrm{h})$ & 10 & 1.77 & -0.006 & 0.003 & 0.08 \\
\hline & BellaAg ${ }^{4}$ & Temperature $\left({ }^{\circ} \mathrm{C}\right)$ & 18 & 0.59 & 1.67 & 0.93 & 0.09 \\
\hline & IceQube & Steps (no.) & 18 & 1.32 & $-4 \times 10^{-5}$ & $1 \times 10^{-5}$ & $<0.05$ \\
\hline & IceQube & Motion index $(-)$ & 18 & 1.28 & $-2 \times 10^{-6}$ & $8 \times 10^{-7}$ & $<0.05$ \\
\hline \multirow[t]{2}{*}{ Autocorrelation } & BellaAg & Temperature $\left({ }^{\circ} \mathrm{C}\right)$ & 18 & 0.01 & 1.39 & 0.71 & 0.07 \\
\hline & IceQube & Lying time $(\min / 15 \mathrm{~min})$ & 18 & 0.27 & 1.46 & 0.65 & $<0.05$ \\
\hline \multirow[t]{3}{*}{ Nonperiodicity } & SensOor & Eating $(\min / 15 \mathrm{~min})$ & 10 & -0.33 & 37.10 & 10.60 & $<0.05$ \\
\hline & IceQube & Lying time $(\min / 15 \mathrm{~min})$ & 18 & 0.03 & 18.50 & 8.22 & $<0.05$ \\
\hline & IceQube & Steps (no.) & 18 & 0.006 & 20.60 & 11.30 & 0.09 \\
\hline
\end{tabular}

\footnotetext{
${ }^{1} \mathrm{TDS}=$ total deficit score.

${ }^{2}$ Agis Automatisering (Harmelen, the Netherlands).

${ }^{3}$ IceRobotics (Edinburgh, UK).

${ }^{4}$ Wynnstay (Powys, UK).
} 


\section{Relation Between (Dynamic) Indicators and TDS Within Farm Context}

Circadian misalignment, sleep deprivation, and exposure to light at night are thought to be primary sources of disruption in different species (especially human shift workers) contributing to adverse health effects (Evans and Davidson, 2013). External factors causing variation between individual circadian patterns within a herd of cows may be related to competition, overstocking, lack of synchronization possibilities due to housing facilities, or ambiguity in the ranking order due to the frequent change in herd composition (Cooper et al., 2007; Collings et al., 2011; Witaifi et al., 2018). When overstocking leads to deprivation of lying time, a rescheduling of the daily time budget may occur to recover the lost lying time (Cooper et al., 2007). This may explain the variation in disturbed circadian patterns that we found in the current study, although recently no relations were found between behavioral circadian patterns and stocking density (Wang et al., 2016). Group size, grouping strategy, and group feeding behavior may have a large effect on the competition between animals for feed, feed intake, and resting space (Grant and Albright, 1995). Aggressive interactions at the feed bunk or avoiding aggressive interactions have previously been found to be related to the development of metritis after calving (Huzzey et al., 2007). In addition, physiology is influenced by overstocking (Huzzey et al., 2012). Competition itself is also perceived differently depending on the social rank degree of the cow (Sundrum, 2015). Under high stocking densities, the behavior of certain cows, possibly the more submissive ones (Huzzey et al., 2006), may have been compromised to the extent that diurnal behavior patterns were disturbed and the intake of high-quality feed was inadequate (Huzzey et al., 2006). Notably, during the risk assessment analysis CowCompas (Koekompas), overstocking in combination with insufficient access to important resources such as feed, water, and cubicles was identified as an important risk factor at the farm where we performed the study. Thus, we suggest that cows with regular circadian patterns and low nonperiodicities were more successful in coping with the competitive situation at the farm where our experiment took place than cows with irregular circadian rhythms and high nonperiodicities. Such differences between cows, in turn, ultimately may have led to differences in TDS after calving. If we define resilience in animals as the capacity to cope with perturbations in their environment and return rapidly to their prechallenge status (Colditz and Hine, 2016), cows with regular circadian patterns could be considered as animals showing high resilience on this farm. Future research is necessary to address the specific hy- pothesis that avoiding risk factors such as overstocking or insufficient access to important resources (e.g., feed or water) during the transition period in dairy cows leads to a decrease in nonperiodicity of behavioral and physiological measures of all cows before calving and a concomitant decrease in TDS after calving.

\section{Dynamic Indicators of Resilience}

Changing dynamics, expressed by increasing autocorrelation and variance, have been proposed as indicators for resilience in a variety of complex dynamic systems (Scheffer et al., 2009). Resilience in general is defined as the capacity of a system to absorb disturbance and reorganize while undergoing change in order to retain essentially the same function, structure, and feedback and therefore identity (Ge et al., 2016). When the capacity to adjust fails, the system will collapse or shift to a different state or equilibrium, also referred to as tipping point (Scheffer et al., 2009). Once the threshold is passed, intrinsic processes in the system form a positive feedback loop, leading to a shift in state toward an alternative equilibrium (van Nes et al., 2016). Close to a tipping point, the state dynamics become increasingly slow in recovering from small perturbations, a phenomenon known in dynamical systems theory as critical slowing down (Scheffer et al., 2009). As a consequence of this slowing down, its dynamics in a stochastic environment are characterized by increasing standard deviation or variance and increasing correlation between subsequent states (Scheffer et al., 2009). We defined resilience in dairy cows as the capability to stay healthy during and after the demanding challenge of transition by adapting the definition of resilience in farm animals as proposed by Colditz and Hine (2016). When the cow is not able to adequately reorganize, critical slowing down may be clinically revealed as periparturient disorder. The self-propelled accelerating change (positive feedback loop) causing a tipping point as described by van Nes et al. (2016) also occurs in cows that cannot adequately handle the transition phase. The ability to self-recover is lost in this case, and intervention with medications is needed to help the animal recover. The positive feedback loop can be initiated at the moment when the gap between nutrient intake and demand becomes too large. Metabolic disorders will appear and may lead to further health problems, such as infections through dysfunctional inflammatory responses and oxidative stress (Sundrum, 2015). This will reduce DMI, reinforcing the metabolic stress, which will further increase the dysfunctional inflammatory responses, and, hence, the positive feedback loop is in progress. Conversely, postpartum infections can also start the positive feedback loop. Collectively, the present findings show that dy- 
namic properties of sensor data are of great additional value and seem to fit theoretical concepts of resilience. We suggest that dynamic indicators of physiological parameters such as increased nonperiodicity, variance, and autocorrelations in ear and rumen temperature are signs of an upcoming tipping point between a healthy state and a clinically detectable disease state and may represent dynamic indicators for individual resilience of cows.

\section{Multivariate Analysis Versus Single Predictor Model}

Combining average eating time, variance in ear temperature, and nonperiodicity of number of steps exposed the lowest prediction error for TDS. A possible reason why best subset selection results in a model with multiple predictors, representing static as well as dynamic signal properties, might be because such a combination of predictors better reflects the numerous factors and interconnected risk factors that are involved in the complex pathophysiology of postpartum diseases (Sundrum, 2015). However, given the low numbers of observations in the present experiment, our findings have to be treated with care. Prediction accuracy for TDS increased when static as well as dynamic signal properties of IceQube sensors were used but decreased when all sensors were combined, using only 7 cows in the analysis. Perhaps the multivariate approach explored here might lead to novel ways of studying the complexity of adaptive processes in cows during the transition period.

\section{CONCLUSIONS}

This case study presents statistical evidence that static and dynamic aspects of continuously recorded high-resolution physiological and behavioral measures before parturition can predict disease severity in cows during the early-lactation period. Being able to identify in a timely manner cows at risk for the development of disease after calving would be extremely helpful because it would allow the farmer to anticipate potential health problems and to take remedial action if required. Statistically, the most optimal linear combination of predictors to predict disease severity during the earlylactation period consisted of low average eating time, nonperiodicity in number of steps (indicative of disturbance of daily patterns), and variance in ear temperature. Our results suggest that these quantitative variables derived from sensor data are promising indicators for disease severity or resilience during the transition period. Follow-up studies with more cows and on different farms are required to extend and confirm the present findings.

\section{ACKNOWLEDGMENTS}

We thank the staff of the Veterinair Kennis Centrum Oost Nederland (VKON; Den Ham, the Netherlands) and veterinarians of Dierenkliniek Den Ham (Den Ham, the Netherlands) for their valuable assistance with the coordination and implementation of the experiment and for performing the clinical assessments. We also thank Joost de Veer (Texel, the Netherlands) and Gerrit Hegen (Sleen, the Netherlands) for carrying out KoeKompas and for their help and inspiration throughout the study, and Gerrit Braakman and Remco de Veer of AgroVision (Deventer, the Netherlands) for fruitful discussions during the course of the research. We are especially grateful to the farmer family (Overijssel, the Netherlands) for their hospitality and practical support and for generously providing access to their farm during the experiment. The study was funded by the province of Overijssel, the Netherlands, and the Dutch Melkveefonds (the Netherlands).

\section{REFERENCES}

Bewley, J. M., and M. M. Schutz. 2010. Recent studies using a reticular bolus system for monitoring dairy cattle core body temperature. Pages 218-219 in The First North American Conference on Precision Dairy Management. Toronto, Canada. http://www .precisiondairy.com/proceedings/s11bewley.pdf.

Bikker, J. P., H. van Laar, P. Rump, J. Doorenbos, K. van Meurs, G. M. Griffioen, and J. Dijkstra. 2014. Technical note: Evaluation of an ear-attached movement sensor to record cow feeding behavior and activity. J. Dairy Sci. 97:2974-2979.

Borchers, M. R., Y. M. Chang, I. C. Tsai, B. A. Wadsworth, and J. M. Bewley. 2016. A validation of technologies monitoring dairy cow feeding, ruminating, and lying behaviors. J. Dairy Sci. 99:7458 7466.

Calamari, L., N. Soriani, G. Panella, F. Petrera, A. Minuti, and E. Trevisi. 2014. Rumination time around calving: An early signal to detect cows at greater risk of disease. J. Dairy Sci. 97:3635-3647.

Colditz, I. G., and B. C. Hine. 2016. Resilience in farm animals: Biology, management, breeding and implications for animal welfare. Anim. Prod. Sci. 56:1961-1983.

Collings, L. K. M., D. M. Weary, N. Chapinal, and M. A. G. von Keyserlingk. 2011. Temporal feed restriction and overstocking increase competition for feed by dairy cattle. J. Dairy Sci. 94:5480-5486.

Cooper, M. D., D. R. Arney, and C. J. C. Phillips. 2007. Two- or fourhour lying deprivation on the behavior of lactating dairy cows. J. Dairy Sci. 90:1149-1158.

Davidson, A. J., M. T. Sellix, J. Daniel, S. Yamazaki, M. Menaker, and G. D. Block. 2006. Chronic jet-lag increases mortality in aged mice. Curr. Biol. 16:R914-R916.

de Mol, R. M., G. André, E. J. B. Bleumer, J. T. N. van der Werf, Y. de Haas, and C. G. van Reenen. 2013. Applicability of day-to-day variation in behavior for the automated detection of lameness in dairy cows. J. Dairy Sci. 96:3703-3712.

Edmonson, A. J., I. J. Lean, L. D. Weaver, T. Farver, and G. Webster. 1989. A body condition scoring chart for Holstein dairy cows. J. Dairy Sci. 72:68-78.

Evans, J. A., and A. J. Davidson. 2013. Health consequences of circadian disruption in humans and animal models. Pages 283-323 in Progress in Molecular Biology and Translational Science. Vol. 119. U. G. Martha, ed. Academic Press, Wageningen, the Netherlands.

Ge, L., N. P. R. Anten, I. D. E. van Dixhoorn, P. H. Feindt, K. Kramer, R. Leemans, M. P. M. Meuwissen, H. Spoolder, and W. 
Sukkel. 2016. Why we need resilience thinking to meet societal challenges in bio-based production systems. Curr. Opin. Environ. Sustain. 23:17-27.

Gillette, M. U. 2013. Introduction to biological timing in health and disease. Pages xi-xvi in Progress in Molecular Biology and Translational Science. Vol. 119. U. G. Martha, ed. Academic Press, Wageningen, the Netherlands.

Goldhawk, C., N. Chapinal, D. M. Veira, D. M. Weary, and M. A. G. von Keyserlingk. 2009. Prepartum feeding behavior is an early indicator of subclinical ketosis. J. Dairy Sci. 92:4971-4977.

Grant, R. J., and J. L. Albright. 1995. Feeding behavior and management factors during the transition period in dairy cattle. J. Anim. Sci. 73:2791-2803.

Grummer, R. R. 1995. Impact of changes in organic nutrient metabolism on feeding the transition dairy cow. J. Anim. Sci. 73:28202833.

Hajer, R., J. Hendrujse, L. J. E. Rutgers, M. M. Sloet van Oldruitenborgh-Oosterbaan, and G. C. van der Weijden. 1988. Het klinisch onderzoek bij grote huisdieren. Wetenschappelijke uitgeverij Bunge, Utrecht, the Netherlands.

Hulsen, J. 2012. Cow Signals: A Practical Guide for Dairy Farm Management. Roodbont, Zutphen, the Netherlands.

Huzzey, J. M., T. J. DeVries, P. Valois, and M. A. G. Von Keyserlingk. 2006. Stocking density and feed barrier design affect the feeding and social behavior of dairy cattle. J. Dairy Sci. 89:126-133.

Huzzey, J. M., T. F. Duffield, S. J. LeBlanc, D. M. Veira, D. M. Weary, and M. A. G. von Keyserlingk. 2009. Short communication: Haptoglobin as an early indicator of metritis. J. Dairy Sci. 92:621-625.

Huzzey, J. M., S. Mann, D. V. Nydam, R. J. Grant, and T. R. Overton. 2015. Associations of peripartum markers of stress and inflammation with milk yield and reproductive performance in Holstein dairy cows. Prev. Vet. Med. 120:291-297.

Huzzey, J. M., D. V. Nydam, R. J. Grant, and T. R. Overton. 2011. Associations of prepartum plasma cortisol, haptoglobin, fecal cortisol metabolites, and nonesterified fatty acids with postpartum health status in Holstein dairy cows. J. Dairy Sci. 94:5878-5889.

Huzzey, J. M., D. V. Nydam, R. J. Grant, and T. R. Overton. 2012. The effects of overstocking Holstein dairy cattle during the dry period on cortisol secretion and energy metabolism. J. Dairy Sci. 95:4421-4433.

Huzzey, J. M., D. M. Veira, D. M. Weary, and M. A. G. Von Keyserlingk. 2007. Prepartum behavior and dry matter intake identify dairy cows at risk for metritis. J. Dairy Sci. 90:3220-3233.

James, G., D. Witten, T. Hastie, and R. Tibshirani. 2013. An Introduction to Statistical Learning: With Applications in R. Springer Science and Business Media, New York, NY.

LeBlanc, S. J., K. D. Lissemore, D. F. Kelton, T. F. Duffield, and K. E. Leslie. 2006. Major advances in disease prevention in dairy cattle. J. Dairy Sci. 89:1267-1279.

Mulligan, F. J., and M. L. Doherty. 2008. Production diseases of the transition cow. Vet. J. 176:3-9.

Munksgaard, L., C. Reenen, and R. Boyce. 2006. Automatic monitoring of lying, standing and walking behavior in dairy cattle. J. Anim. Sci. 84(Suppl.).

Peng, C. K., M. Costa, and A. L. Goldberger. 2009. Adaptive data analysis of complex fluctuations in physiologic time series. Adv. Adapt. Data Anal. 1:61-70.
Pereira, G. M., B. J. Heins, and M. I. Endres. 2018. Technical note: Validation of an ear-tag accelerometer sensor to determine rumination, eating, and activity behaviors of grazing dairy cattle. J. Dairy Sci. 101:2492-2495.

R Core Team. 2017. R: A language and environment for statistical computing. R Foundation for Statistical Computing, Vienna, Austria. https://www.R-project.org/.

Roberts, T., N. Chapinal, S. J. LeBlanc, D. F. Kelton, J. Dubuc, and T. F. Duffield. 2012. Metabolic parameters in transition cows as indicators for early-lactation culling risk. J. Dairy Sci. 95:30573063.

Rutten, C. J., C. Kamphuis, H. Hogeveen, K. Huijps, M. Nielen, and W. Steeneveld. 2017. Sensor data on cow activity, rumination, and ear temperature improve prediction of the start of calving in dairy cows. Comput. Electron. Agric. 132:108-118.

Scheffer, M., J. Bascompte, W. A. Brock, V. Brovkin, S. R. Carpenter, V. Dakos, H. Held, E. H. Van Nes, M. Rietkerk, and G. Sugihara. 2009. Early-warning signals for critical transitions. Nature 461:53-59.

Schirmann, K., N. Chapinal, D. M. Weary, L. Vickers, and M. A G. Von Keyserlingk. 2013. Short communication: Rumination and feeding behavior before and after calving in dairy cows. J. Dairy Sci. 96:7088-7092.

Schirmann, K., D. M. Weary, W. Heuwieser, N. Chapinal, R. L. A Cerri, and M. A. G. von Keyserlingk. 2016. Short communication: Rumination and feeding behaviors differ between healthy and sick dairy cows during the transition period. J. Dairy Sci 99:9917-9924

Seifi, H. A., S. J. LeBlanc, K. E. Leslie, and T. F. Duffield. 2011 Metabolic predictors of post-partum disease and culling risk in dairy cattle. Vet. J. 188:216-220.

Sundrum, A. 2015. Metabolic disorders in the transition period indicate that the dairy cows' ability to adapt is overstressed. Animals (Basel) 5:978-1020.

Timsit, E., S. Assié, R. Quiniou, H. Seegers, and N. Bareille. 2011. Early detection of bovine respiratory disease in young bulls using reticulo-rumen temperature boluses. Vet. J. 190:136-142.

Trevisi, E., M. Amadori, S. Cogrossi, E. Razzuoli, and G. Bertoni 2012. Metabolic stress and inflammatory response in high-yielding, periparturient dairy cows. Res. Vet. Sci. 93:695-704.

Urton, G., M. A. G. von Keyserlingk, and D. M. Weary. 2005. Feeding behavior identifies dairy cows at risk for metritis. J. Dairy Sci 88:2843-2849

van Nes, E. H., B. M. S. Arani, A. Staal, B. van der Bolt, B. M. Flores, S. Bathiany, and M. Scheffer. 2016. What do you mean, "tipping point"? Trends Ecol. Evol. 31:902-904.

Wang, F. X., D. F. Shao, S. L. Li, Y. J. Wang, A. Azarfar, and Z. J. Cao. 2016. Effects of stocking density on behavior, productivity, and comfort indices of lactating dairy cows. J. Dairy Sci. 99:37093717 .

Weary, D. M., J. M. Huzzey, and M. A. G. Von Keyserlingk. 2009. Board-invited review: Using behavior to predict and identify ill health in animals. J. Anim. Sci. 87:770-777.

Witaifi, A. A., A. B. A. Ali, and J. M. Siegford. 2018. Stall and feed bunk stocking rates impact cows' diurnal behavior and activity in automatic milking system farms. J. Vet. Behav. 24:48-55. 